Review

\title{
Laccase-catalyzed, Phytochemical-mediated Protein Crosslinking Conjugates
}

\author{
Tsun-Thai Chai ${ }^{1,2}$, Jianbo Xiao ${ }^{3}$, Sharmila Mohana Dass ${ }^{2}$, Fai-Chu Wong ${ }^{1,2, *}$ \\ ${ }^{1}$ Centre for Biodiversity Research, Universiti Tunku Abdul Rahman, Kampar 31900, Malaysia \\ ${ }^{2}$ Biochemistry Program, Department of Chemical Science, Faculty of Science, Universiti Tunku Abdul Rahman, Kampar 31900, Malaysia \\ ${ }^{3}$ Institute of Chinese Medical Sciences, SKL of Quality Research in Chinese Medicine, University of Macau, Avenida da Universidade, Taipa, Macau, China
}

\section{ARTICLE INFO}

\section{Article History}

Received 14 January 2020

Accepted 15 February 2020

\section{Keywords}

Crosslinking

enzyme

ferulic acid

laccase

milk

phytochemical

protein

\begin{abstract}
Laccase is a Class 1 oxidoreductase enzyme, which catalyzes the oxidation of a wide range of phenolic substrates. Previously, several reviews had highlighted on the industrial importance of laccase, including in food industry. Similarly, the ability of laccase to crosslink and polymerize food protein molecules had also been reviewed. However, no much review information is available on the crosslinking of protein molecules, mediated by phytochemicals. In this review, we highlighted on the laccase-catalyzed, phytochemical-mediated protein crosslinking conjugates, and their associated functional changes, reported by different research groups over the last 10 years. In many of these reported studies, milk protein components are utilized, along with the addition of selected phytochemicals (chlorogenic acid, ferulic acid). As proteins are major dietary components, while phytochemicals are plant-derived chemicals with well-studied bioactivities, their conjugations could potentially lead to the identification of novel health-promoting compounds.
\end{abstract}

(c) 2020 International Association of Dietetic Nutrition and Safety. Publishing services by Atlantis Press International B.V. This is an open access article distributed under the CC BY-NC 4.0 license (http://creativecommons.org/licenses/by-nc/4.0/).

\section{INTRODUCTION}

Laccases (EC 1.10.3.2) (Figure 1) are a group of oxidoreductase enzymes, with members originate from fungi, plants and bacteria [1]. These copper-containing enzymes catalyze the oxidation of a wide range of phenolic substrates, concurrent with the reductive conversion of oxygen molecules to water molecules. In the process, substrate crosslinking is frequently observed. Because of their broad substrate specificity, laccases are frequently considered as excellent industrial enzymes, with potential applications in various manufacturing and processing steps [2]. Previously, industrial applications of laccases have been widely reported, ranged from food and beverage industry, textiles, pulp and paper processing, to bioremediation [2,3]. Similarly, laccase application in food protein crosslinking had also been reviewed, with the accompanied functional improvement highlighted $[4,5]$. However, despite the numerous reviews available on laccases, limited review information is focused on the progress of laccase-catalyzed protein crosslinking, mediated by different phytochemicals. In this review article, we highlighted on the laccase-catalyzed, phytochemical-mediated protein crosslinking conjugates, along with the associated functional and bioactivity changes, reported by different research groups over the last 10 years.

“Corresponding author. Email: wongfc@utar.edu.my

Peer review under responsibility of the International Association of Dietetic Nutrition and Safety

\section{PHYTOCHEMICALS}

Phytochemicals are diverse groups of chemical compounds, frequently associated with a variety of bioactivities, and derived mainly from members of the plant kingdom. These plant-derived chemical metabolites consist of various derivatives of alkaloids, flavonoids, phenolic acids, and terpenoids [6,7]. In nature, plants reply on these diverse phytochemicals to cope with various threats from the harsh environment, as well as other abiotic and biotic challenges. In traditional and modern medicine, many of these phytochemicals contained in medicinal plant extracts have been treasured for their medicinal values and health-promoting potentials $[7,8]$. Similarly, contemporary scientific research has provided concrete links between selected phytochemicals with various health-promoting and therapeutic bioactivities, ranged from antioxidant, anti-diabetic, anti-proliferative, anti-hypertensive, bacterial inhibition, to cellular protection $[9,10]$. Additionally, proteomics and nutrigenomics studies are trying to link how selected dietary phytochemicals could affect our gene expression, and subsequently bodily health [11]. Frequently, once screened and identified with a health-promoting and disease-fighting bioactivities, these plant-derived phytomolecules are next subjected to additional molecular modifications and substitutions, in the efforts to generate molecular derivatives with enhanced bioactivity and bioavailability. After successful clinical trials, some of these phytochemical derivatives have eventually be developed into commercial therapeutic drugs [12]. In fact, it is estimated that more than half 


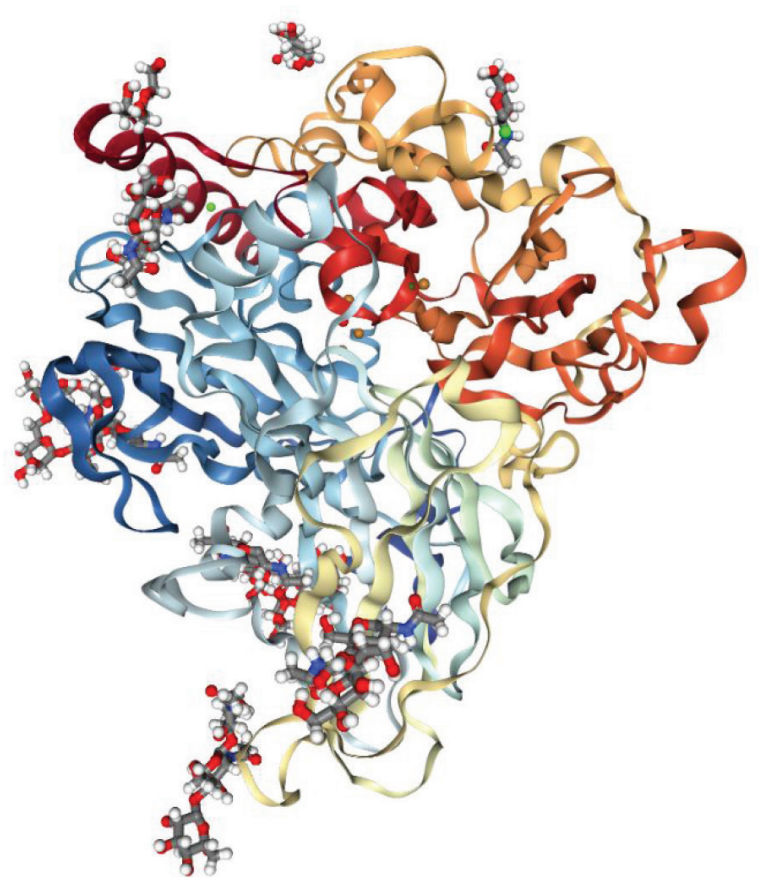

Figure 1 Crystal structure of laccase from Myceliophthora thermophile (PDB ID: 6F5K). Article source: Ernst HA, Jørgensen LJ, Bukh C, Piontek K, Plattner DA, et al. A comparative structural analysis of the surface properties of asco-laccases. PLoS One 2018;13:e206589.

of our modern drugs can trace their origins to plant-derived phytochemicals. Some well-known examples include anti-tumor drugs (vincristine, vinblastine and paclitaxel) and anti-malarial drug (artemisinin), which have previously been reviewed [12].

\section{ALTERATIONS OF PROTEIN STRUCTURAL AND FUNCTIONAL PROPERTIES}

Proteins, as well as peptides and amino acids, represent the major components in dietary food matrix. Numerous research interests have been focused on how these protein compositions will influence the taste, nutritional, as well as the texture and functionality of food. When subjected to thermal treatments and non-enzymatic Maillard reaction, the protein structural compositions will be denatured and altered, frequently accompanied by the formation of new conjugates, with altered tastes and functionalities $[13,14]$. For instance, previous studies had reported on the formation of new amino acid conjugates, following thermal treatments of black garlic and Panax ginseng $[15,16]$. Similarly, hydrolytic enzymes (proteases) have been shown to impart changes in proteins' structural, as well as functional and bioactive properties [17]. In this regard, numerous scientific studies have revealed on how members of the hydrolytic enzymes, especially of the protease grouping, could be applied to promote the release of bioactive peptides frequently associated with enhanced and novel bioactivities [18-20]. Likewise, oxidative enzymes (laccase, transglutaminase, tyrosinase) have previously been reported to produce crosslinked proteins with altered functionalities such as strength, gelation, emulsifying and water holding capacities [4,21].
Laccase, being a member of the oxidative enzymes, had previously been utilized to introduce crosslinking into various protein components, and the accompanied alterations in structural and functional properties have been reviewed. Among the various oxidative enzymes capable of protein crosslinking, laccase represents a particularly popular choice, encouraged by its broad substrate specificity and hence potential applications in diverse industries [2,4]. For example, the addition of laccase and sugar beet pectin had been shown to improve the stability and properties of concentrated protein emulsion [22]. Similarly, laccase-mediated crosslinking had helped to improve the rheological properties of amadumbe dough [23]. However, few review information is available on the crosslinking of protein molecules, mediated by phytochemicals. In the subsequent sections of this review, we highlighted on the laccase-catalyzed, phytochemical-mediated protein crosslinking conjugates, reported by various research groups over the last ten years (Table 1). In addition, we also reviewed on the associated structural and functional changes, as well as the various methodologies used to investigate the phytochemical-mediated protein crosslinking conjugates. As proteins are the major dietary components, while phytochemicals are plant-derived chemicals with well-studied bioactivities, their conjugations could potentially lead to the identification of novel health-promoting compounds.

\section{CHARACTERISTICS OF LACCASE- CATALYZED, PHYTOCHEMICAL- MEDIATED PROTEIN CROSSLINKING}

To date, the majority of reported works on laccase-catalyzed, phytochemical-mediated protein crosslinking was found utilizing various milk protein components (casein and lactalbumin) as starting materials (Table 1). This is comprehensible, considering the high protein contents in milk, as well as the interests in its commercial values. As to date, no related research was found using plant-derived protein in laccase-catalyzed, phytochemicalmediated crosslinking study. The lack of related research using plant-derived proteins may due to the relatively low protein contents in plant matrices. As for the enzyme sources, most of the reported studies focused on using laccases originated from fungi and mushrooms. Among these reviewed studies, half of them involved using laccases from Trametes hirsuta and Trametes versicolor $[28,30-33,36]$.

In these reported studies on laccase-catalyzed, phytochemicalmediated protein crosslinking, a variety of phytochemicals (catechin, chlorogenic acid, ferulic acid) have been reported to facilitate the protein crosslinking processes (Table 1). Among these phytochemicals, members of the hydroxycinnamic acids (chlorogenic acid, ferulic acid) received the widest research interest (Figure 2). In fact, among the research studies covered in this review, almost two-thirds of them were performed using ferulic acid. Previously, ferulic acid and its derivatives have been reported with health-promoting potentials, ranged from anti-hypertensive to cellular protection [37,38]. Additionally, a recent study also highlighted on how ferulic acid synergistically enhanced the performance of metformin in an anti-diabetic study [39]. Studies have postulated that phytochemical molecules with smaller sizes may ease their access into the laccase's active site, 


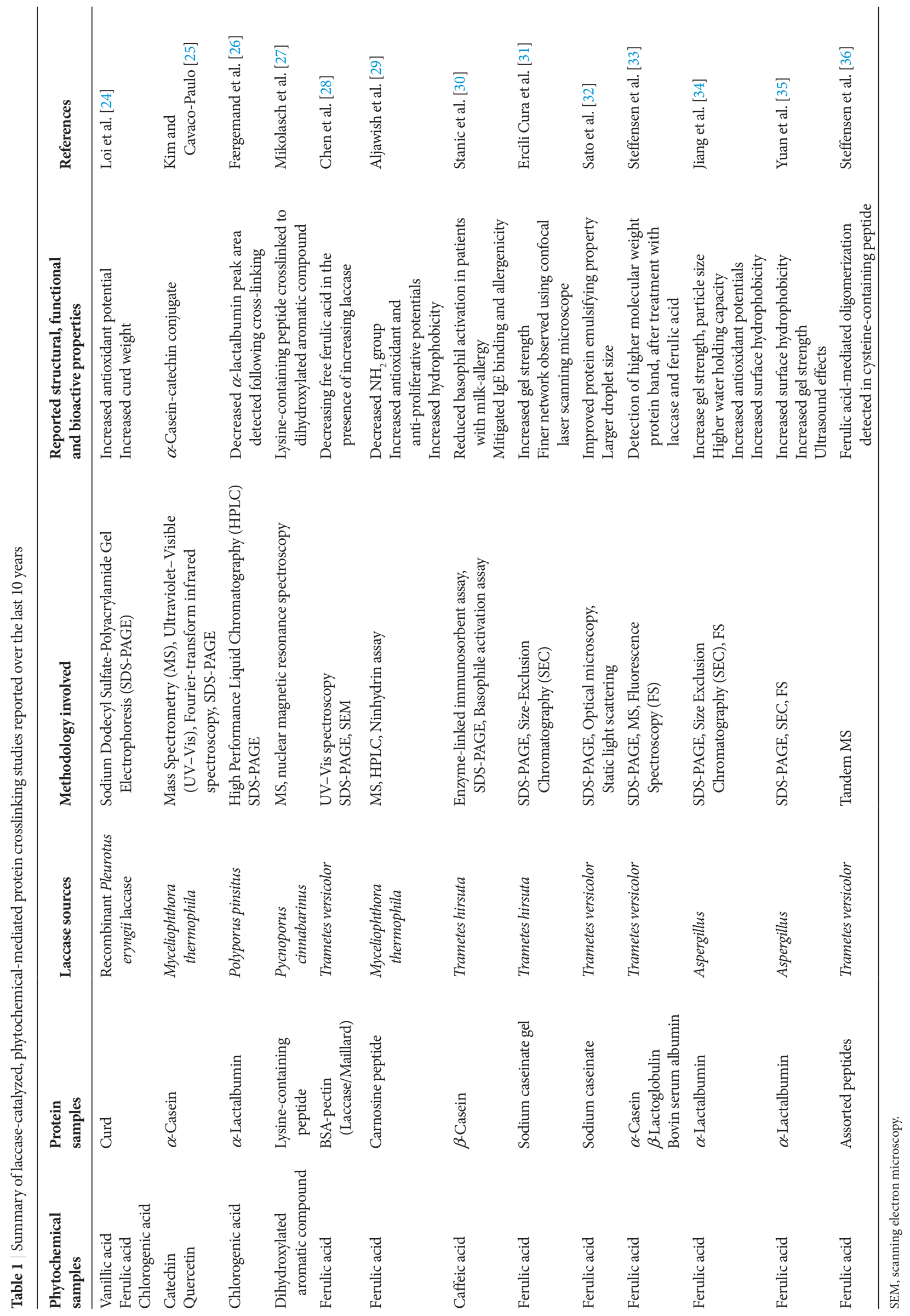




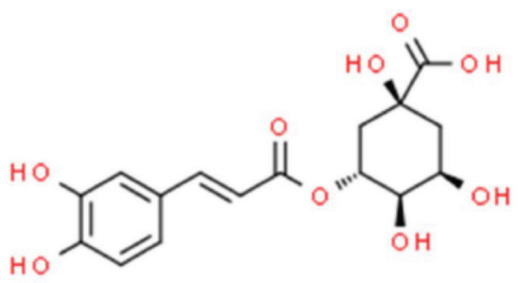

Chlorogenic acid

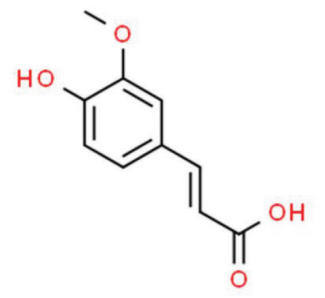

Ferulic acid
Figure 2 Chemical structures of three of the commonly used phytochemicals. Detailed information, including synonyms and chemical properties, of these hydroxycinnamic acids are available at ChemSpider (http://www.chemspider.com/).

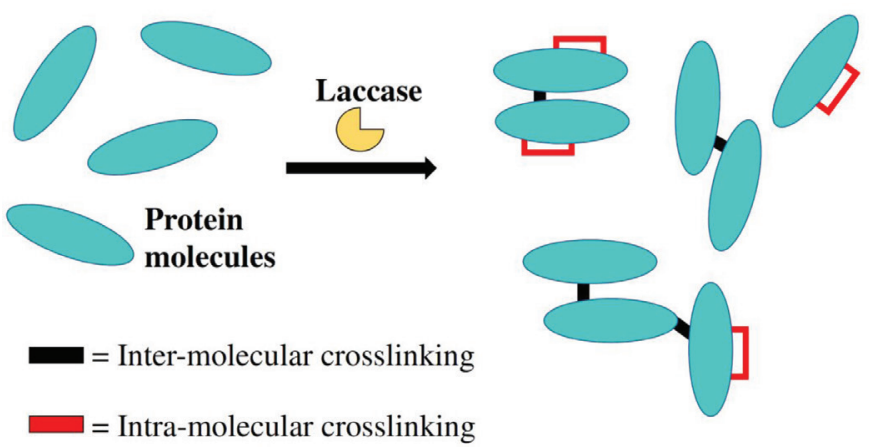

Figure 3 Inter- versus intra-molecular crosslinking in protein molecules.

during the enzymatic catalytic reactions. On the contrary, larger phytochemical molecules may yield steric hindrance that could hinder with their participation in the laccase-catalyzed reactions. These reasons may help to explain the high utilization of ferulic acid in laccase-catalyzed, phytochemical-mediated protein crosslinking.

In the presence of laccase, catalytic reactions were carried out and subsequently led to the formation of covalent bonding between adjacent protein molecules. Here, protein crosslinking was reportedly formed via adjacent tyrosine residues [33]. Similarly, laccasecatalyzed crosslinking had also been reported via cysteine residues, especially in the presence of selected phytochemicals [33,36]. Interestingly, laccase was also found to activate a lysine-containing peptide, and subsequently catalyzed the crosslinking between this lysine-containing peptide and a dihydroxylated aromatic compound, in a study using mass spectrometry and nuclear magnetic resonance analysis [27]. On the other hand, laccase-catalyzed protein crosslinking reactions could theoretically occur via the formation of inter-molecular, as well as intra-molecular crosslinking. That is, the crosslinking could take place between two different protein molecules, or within the same protein molecule, respectively (Figure 3). In this regard, the extent of inter-molecular crosslinking could have a direct effect on the rheological, as well as functional and bioactive properties. However, this phenomenon is not reported by the studies covered in this review. It would be interesting to have more studies investigated on the extent of inter-molecular protein crosslinking, in the presence of selected phytochemicals.

\section{REPORTED PROPERTY CHANGES IN PHYTOCHEMICAL-MEDIATED PROTEIN CROSSLINKING CONJUGATES}

During the laccase-catalyzed crosslinking, new covalent interactions are introduced into the protein matrices. Consequently, the molecular orientation and arrangements in the protein matrices are altered, which may lead to desired changes in their texture, rheological, as well as functional properties. Similarly, with the addition of selected phytochemicals into these laccase-catalyzed protein crosslinking reactions, the crosslinking patterns will be further altered and increased, along with the introduction of novel structural and functional properties. In Table 1, we summarized the changes in functional properties, observed in these phytochemical-mediated, protein crosslinking conjugates.

Following laccase-catalyzed, ferulic acid-mediated protein crosslinking, some of the novel characteristics introduced are structurally-related. Among them, stronger protein gel strength was reported using sodium caseinate gel and $\alpha$-lactalbumin, following treatments with laccase and ferulic acid $[31,34,35]$. This phenomenon is presumably due to the formation of extensive protein crosslinking networks, and it is agreeable with the detection of finer crosslinking networks under microscopic observation [31]. Previous study had suggested on the idea that ferulic acid's small structure may ease its access to laccase's active site and promote the formation of additional protein crosslinking. In addition to stronger gel strength $[31,34,35]$, higher water holding capacity [34], increased surface hydrophobicity [35], as well as improved protein emulsifying property [32] were also reported with these protein crosslinking conjugates. Similarly, in a dairy product research, increased curd weight was reported, following crosslinking treatment with chlorogenic acid and laccase [24]. On a separate note, in a study involving $\alpha$-lactalbumin and ferulic acid, ultrasound treatment was previously reported with the capability to promote both covalent and non-covalent interactions between $\alpha$-lactalbumin protein molecules and aggregates, subsequently leading to changes in functional property of lactalbumin-ferulic acid conjugates [35]. However, the detailed mechanism remains to be determined.

Besides structural and functional alterations, several research studies also reported on enhanced antioxidant potentials in the ferulic acid-mediated, protein crosslinking conjugates [24,29,34]. Here, the observed antioxidant potentials were detected using DPPH and ABTS scavenging assays, as well as ferrous reducing power assay. This observation is comprehensible, as ferulic acid and other members of the hydroxycinnamic acids had previously been recognized for their antioxidant potentials, when tested in assorted antioxidant assays $[37,38]$. In the laccase-catalyzed protein crosslinking, the incorporated ferulic acid molecules may exert its antioxidant potentials and contributed toward the enhanced antioxidant potentials.

In addition to antioxidant potentials, several research groups also reported on enhancements in other bioactivities, following laccase-catalyzed, phytochemical-mediated protein or peptide crosslinking. For instance, ferulic acid-carnosine conjugates was reported with increased anti-proliferative effect [29]. In this study, when tested on Caco-2 cells (colonic endothelial tumor cells) in a neutral red uptake cell viability assay, the laccase-catalyzed ferulic 
acid-carnosine conjugate was detected with higher cytotoxic effect, compared to either ferulic acid or carnosine alone [29]. Likewise, a separate study reported on the potential application of laccasecatalyzed, phytochemical-mediated protein crosslinking to mitigate allergenicity induced by dairy product [30]. In this allergenicity assessment study, caffeic acid-mediated $\beta$-casein crosslinking was reported with decreased IgE binding, as well as reduced basophil activation in patients with cow milk allergy [30].

\section{ANALYTICAL METHODS USED TO STUDY PROTEIN CROSSLINKING CONJUGATES}

During the investigation of laccase-catalyzed, phytochemicalmediated protein crosslinking, assorted analytical and detection techniques were applied. Among these different techniques, denaturing gel electrophoresis [Sodium Dodecyl Sulfate-Polyacrylamide Gel Electrophoresis (SDS-PAGE)] represented the most widely applied techniques used to detect and confirm the formation of crosslinked proteins $[24,25,31,33]$. On these SDS-PAGE, the presence of unique high Molecular-Weight (MW) band is the frequent indication of crosslinked protein formation. In addition, Size Exclusion Chromatography (SEC) was applied to detect for the formation of phytochemical-mediated, protein crosslinking conjugates $[31,34,35]$. Here, the higher MW protein crosslinking conjugates will be excluded from the pores of the porous beads, and hence eluted in a shorter time from the column. Taking advantage of the difference in molecular size, and consequently the shorter elution time, the protein crosslinking conjugates could be detected separately. Similarly, SEC was applied to provide additional evidence as to the participation of phytochemical, such as ferulic acid, in the protein crosslinking [31], whereby free ferulic acid was shown to disappear from the elution, in the presence of laccase.

On the other hand, combination of advanced analytical methods such as Mass Spectrometry (MS) [25,27,29,36], high performance liquid chromatography $[26,29]$, and fluorescence spectroscopy $[33,34,35]$ were also reportedly applied to investigate the laccase-catalyzed, phytochemical-mediated protein crosslinking. For instance, in a tandem MS study, the finding helped to provide supporting evidence as to the functional roles of tyrosine and cysteine residues in the crosslinked mechanism [36]. Similarly, other instrumental methods such as Nuclear Magnetic Resonance (NMR) spectroscopy and Fourier-Transform Infrared Spectroscopy (FT-IR) were also applied to investigate the protein crosslinking conjugates $[25,27]$. Here, the presence of protein crosslinking products could be identified, by taking advantages of their unique fingerprint peaks corresponding to particular functional groups.

\section{CHALLENGES ENCOUNTERED AND POTENTIAL FUTURE STUDY DIRECTIONS}

As highlighted in the preceding sections of this review article, laccase represents a promising enzyme for the production of phytochemical-mediated protein crosslinking conjugates, with encouraging future prospects and novel applications. Additionally, many of these protein crosslinking conjugates had been reported with enhancements in their structural, functional, and bioactive properties. Yet, the full potential of laccase applications in these aspects remains untapped, with many new applications awaiting for discovery. However, many technical challenges remain to be tackled. Likewise, questions pertaining to the characteristics of protein crosslinking conjugates, as well as the detailed catalytic mechanism, remain to be answered.

For instance, in many cases, during the detection of phytochemicalmediated protein crosslinking conjugates, it remains unclear if the crosslinked protein population consisting of dimers, trimers, or other configurations. Similarly, limited study is available to illustrate on the degree of phytochemical's contributions toward protein crosslinking, in other words, how many of these protein crosslinking conjugates would not be formed in the absence of the aforementioned phytochemicals. In addition, the extents of intra-molecular versus inter-molecular protein crosslinking remain to be characterized (Figure 3). Surely, the formation of crosslinking within the same protein molecule versus between two different protein molecules, respectively, will affect differently on the rheological, functional and bioactive properties of the crosslinked conjugates. Further investigation into this aspect may likely contribute toward our understanding to direct the laccase's crosslinking processes into producing protein crosslinking conjugates with the desired texture and functional properties.

In terms of the catalytic mechanism, the current available literature suggesting that the laccase-catalyzed protein crosslinking is formed via amino residues such as tyrosine, cysteine and lysine $[27,36]$. However, the possibility of laccase enzyme to activate other amino residues could not be ruled out, especially considering the fact that laccase is widely described as an enzyme with broad substrate specificity. This is especially so for amino residues bearing structural similarities to tyrosine and lysine, such as phenylalanine and arginine, respectively. On the other hand, in terms of laccase sources, the majority of the reviewed studies were conducted using native laccase from a variety of fungal sources (Table 1). Among these reviewed research studies, only one reported the use of recombinant laccase from Pleurotus eryngii [24]. Likewise, two-third of these reported laccase-catalyzed, phytochemical-mediated protein crosslinking involved using ferulic acid, a phytochemical from the hydroxycinnamic acid group.

In the future studies, it would be exciting for new laccase sources to be reported. Additionally, it is hoped that current advances in recombinant DNA technology, such as gene shuffling and other enzyme engineering techniques, could be applied to generate new laccase variants with modified substrate specificity, which could activate novel substrates during the protein crosslinking process. These new laccase variants, both recombined and natural sourced, will surely contribute toward the generation of novel protein crosslinking conjugates that are accompanied by novel texture, functionality and bioactive properties. However, these recombinant laccases may result in additional technical challenges awaiting to be solved. Possible challenges that may arise include improper enzyme folding issues which may lead to complications such as denaturation, as well as enzyme solubility issues. Additionally, the recombinant efforts may adversely affect the laccase's active site and yield recombined laccase variants with compromised catalytic efficiency. On a closely related topic, toxicological and pharmacological information 
is currently lacking on protein crosslinking conjugates formed by laccase-catalyzed, phytochemical-mediated reactions. It still remains unclear how these protein crosslinking conjugates will be adsorbed, metabolized, distributed and excreted in an organism. In the future, it would be of great benefits to have research studies reporting on the toxicological and pharmacological aspects of these protein crosslinking conjugates, especially with the applications of the aforementioned recombinant laccases.

\section{CONCLUSION}

In conclusion, laccase-catalyzed, phytochemical-mediated protein crosslinking represents an exciting field to investigate for novel protein crosslinking conjugates that may accompanied by novel structural and functional properties. Similarly, the incorporation of selected phytochemicals with desired bioactive properties, may help to generate protein crosslinking conjugates with novel therapeutic values. In the future, with the investigation of new laccase variants, as well as new protein and phytochemical sources, it is hoped that more phytochemical-mediated, protein crosslinking conjugates will be reported with new functional and bioactive properties.

\section{CONFLICTS OF INTEREST}

The authors declare they have no conflicts of interest.

\section{AUTHORS' CONTRIBUTION}

FCW, TTC and JBX contributed in writing, drafting and preparing the manuscript. SMD contributed in writing and preparing figures for the manuscript.

\section{ACKNOWLEDGMENT}

We would like to extend our gratitude to funding support from UTAR Research Fund (UTARRF).

\section{ABBREVIATIONS}

ABTS, 2,2'-azino-bis-3-ethylbenzothiazoline-6-sulfonic acid; DPPH, 2,2-diphenyl-1-picrylhydrazyl; ELISA, enzyme-linked immunosorbent assay; FS, fluorescence spectroscopy; FT-IR, Fourier-transform infrared spectroscopy; HPLC, high performance liquid chromatography; MS, mass spectrometry; MW, molecular-weight; NMR, nuclear magnetic resonance; SDS-PAGE, sodium dodecyl sulfate-polyacrylamide gel electrophoresis; SEC, size exclusion chromatography; UV-Vis, ultraviolet-visible.

\section{REFERENCES}

[1] Mattinen ML, Hellman M, Permi P, Autio K, Kalkkinen N, Buchert J. Effect of protein structure on laccase-catalyzed protein oligomerization. J Agric Food Chem 2006;54:8883-90.
[2] Martínez AT, Ruiz-Dueñas FJ, Camarero S, Serrano A, Linde D, Lund $\mathrm{H}$, et al. Oxidoreductases on their way to industrial biotransformations. Biotechnol Adv 2017;35:815-31.

[3] Mate DM, Alcalde M. Laccase: a multi-purpose biocatalyst at the forefront of biotechnology. Microb Biotechnol 2017;10:1457-67.

[4] Isaschar-Ovdat S, Fishman A. Crosslinking of food proteins mediated by oxidative enzymes - a review. Trends Food Sci Technol 2018;72:134-43.

[5] Buchert J, Cura DE, Ma H, Gasparetti C, Monogioudi E, Faccio G, et al. Crosslinking food proteins for improved functionality. Annu Rev Food Sci Technol 2010;1:113-38.

[6] Schnekenburger M, Dicato M, Diederich M. Plant-derived epigenetic modulators for cancer treatment and prevention. Biotechnol Adv 2014;32:1123-32.

[7] Cao H, Chai TT, Wang X, Morais-Braga MFB, Yang JH, Wong FC, et al. Phytochemicals from fern species: potential for medicine applications. Phytochem Rev 2017;16:379-440.

[8] Chai TT, Wong FC, Abd Manan F, Ooh KF, Mohd Ismail NI. Orthosiphon aristatus: a review of traditional uses, phytochemical profile, and pharmacological properties. Tradit Folk Herb Med Recent Res 2014;2:153-87.

[9] Xiao JB, Bai WB. Bioactive phytochemicals. Crit Rev Food Sci Nutr 2019;59:827-9.

[10] Xiao JB, Hogger P. Dietary polyphenols and type 2 diabetes: current insights and future perspectives. Curr Med Chem 2015;22:23-38.

[11] Santos BS, Silva LCN, Silva TD, Rodrigues JFS, Grisotto MAG, Correia MTS, et al. Application of omics technologies for evaluation of antibacterial mechanisms of action of plant-derived products. Front Microbiol 2016;7:1466.

[12] Wong FC, Tan ST, Chai TT. Phytochemical-mediated protein expression profiling and the potential applications in therapeutic drug target identifications. Crit Rev Food Sci Nutr 2016;56: S162-S70.

[13] Zhao C, Liu Y, Lai S, Cao H, Guan Y, Cheang WS, et al. Effects of domestic cooking process on the chemical and biological properties of dietary phytochemicals. Trends Food Sci Technol 2019;85:55-66.

[14] Wong FC, Chai TT, Xiao JB. The influences of thermal processing on phytochemicals and possible routes to the discovery of new phytochemical conjugates. Crit Rev Food Sci Nutr 2019;59:947-52.

[15] Ríos-Ríos KL, Vázquez-Barrios ME, Gaytán-Martínez M, Olano A, Montilla A, Villamiel M. 2-Furoylmethyl amino acids as indicators of Maillard reaction during the elaboration of black garlic. Food Chem 2018;240:1106-12.

[16] Cho EJ, Piao XL, Jang MH, Baek SH, Kim HY, Kang KS, et al. The effect of steaming on the free amino acid contents and antioxidant activity of Panax ginseng. Food Chem 2008;107:876-82.

[17] Chai TT, Tan YN, Ee KY, Xiao JB, Wong FC. Seeds, fermented foods, and agricultural by-products as sources of plant-derived antibacterial peptides. Crit Rev Food Sci Nutr 2019;59:S162-S77.

[18] Wong FC, Xiao J, Ong MGL, Pang MJ, Wong SJ, Teh LK, et al. Identification and characterization of antioxidant peptides from hydrolysate of blue-spotted stingray and their stability against thermal, $\mathrm{pH}$ and simulated gastrointestinal digestion treatments. Food Chem 2019;271:614-22.

[19] Zhang L, Zhao GX, Zhao YQ, Qiu YT, Chi CF, Wang B. Identification and active evaluation of antioxidant peptides from protein hydrolysates of Skipjack Tuna (Katsuwonus pelamis) head. Antioxidants 2019;8:318. 
[20] Sheng J, Yang X, Chen J, Peng T, Yin X, Liu W, et al. Antioxidative effects and mechanism study of bioactive peptides from defatted walnut (Juglans regia L.) meal hydrolysate. J Agric Food Chem 2019;67:3305-12.

[21] Heck T, Faccio G, Richter M, Thöny-Meyer L. Enzymecatalyzed protein crosslinking. Appl Microbiol Biotechnol 2013;97:461-75.

[22] Chen P, Chen W, Jiang S, Zhong Q, Chen H, Chen W. Synergistic effect of laccase and sugar beet pectin on the properties of concentrated protein emulsions and its application in concentrated coconut milk. Molecules 2018;23:pii: E2591.

[23] Manhivi VE, Amonsou EO, Kudanga T. Laccase-mediated crosslinking of gluten-free amadumbe flour improves rheological properties. Food Chem 2018;264:157-63.

[24] Loi M, Quintieri L, Fanelli F, Caputo L, Mulè G. Application of a recombinant laccase-chlorogenic acid system in protein crosslink and antioxidant properties of the curd. Food Res Int 2018;106:763-70.

[25] Kim S, Cavaco-Paulo A. Laccase-catalysed protein-flavonoid conjugates for flax fibre modification. Appl Microbiol Biotechnol 2012;93:585-600.

[26] Færgemand M, Otte J, Qvist KB. Cross-linking of whey proteins by enzymatic oxidation. J Agric Food Chem 1998;46:1326-33.

[27] Mikolasch A, Hahn V, Manda K, Pump J, Illas N, Gördes D, et al. Laccase-catalyzed cross-linking of amino acids and peptides with dihydroxylated aromatic compounds. Amino Acids 2010;39: 671-83.

[28] Chen H, Ji A, Qiu S, Liu Y, Zhu Q, Yin L. Covalent conjugation of bovine serum album and sugar beet pectin through Maillard reaction/laccase catalysis to improve the emulsifying properties. Food Hydrocoll 2018;76:173-83.

[29] Aljawish A, Chevalot I, Madad N, Paris C, Muniglia L. Laccase mediated-synthesis of hydroxycinnamoyl-peptide from ferulic acid and carnosine. J Biotechnol 2016;227:83-93.
[30] Stanic D, Monogioudi E, Dilek E, Radosavljevic J, AtanaskovicMarkovic M, Vuckovic O, et al. Digestibility and allergenicity assessment of enzymatically crosslinked $\beta$-casein. Mol Nutr Food Res 2010;54:1273-84.

[31] Ercili Cura D, Lantto R, Lille M, Andberg M, Kruus K, Buchert J. Laccase-aided protein modification: effects on the structural properties of acidified sodium caseinate gels. Int Dairy J 2009;19:737-45.

[32] Sato ACK, Perrechil FA, Costa AAS, Santana RC, Cunha RL. Cross-linking proteins by laccase: effects on the droplet size and rheology of emulsions stabilized by sodium caseinate. Food Res Int 2015;75:244-51.

[33] Steffensen CL, Andersen ML, Degn PE, Nielsen JH. Crosslinking proteins by laccase-catalyzed oxidation: importance relative to other modifications. J Agric Food Chem 2008; 56:12002-10.

[34] Jiang Z, Yuan X, Yao K, Li X, Zhang X, Mu Z, et al. Laccase-aided modification: effects on structure, gel properties and antioxidant activities of $\alpha$-lactalbumin. LWT 2017;80:355-63.

[35] Yuan XY, Li XY, Zhang XL, Mu ZS, Gao ZL, Jiang LZ, et al. Effect of ultrasound on structure and functional properties of laccase-catalyzed $\alpha$-lactalbumin. J Food Eng 2018;223:116-23.

[36] Steffensen CL, Stensballe A, Kidmose U, Degn PE, Andersen ML, Nielsen JH. Modifications of amino acids during ferulic acidmediated, laccase-catalysed cross-linking of peptides. Free Radic Res 2009;43:1167-78.

[37] de Paiva LB, Goldbeck R, dos Santos WD, Squina FM. Ferulic acid and derivatives: molecules with potential application in the pharmaceutical field. Braz J Pharm Sci 2013;49:395-411.

[38] Alam MA. Anti-hypertensive effect of cereal antioxidant ferulic acid and its mechanism of action. Front Nutr 2019;6:121.

[39] Nankar R, Prabhakar PK, Doble M. Hybrid drug combination: combination of ferulic acid and metformin as anti-diabetic therapy. Phytomedicine 2017;37:10-13. 